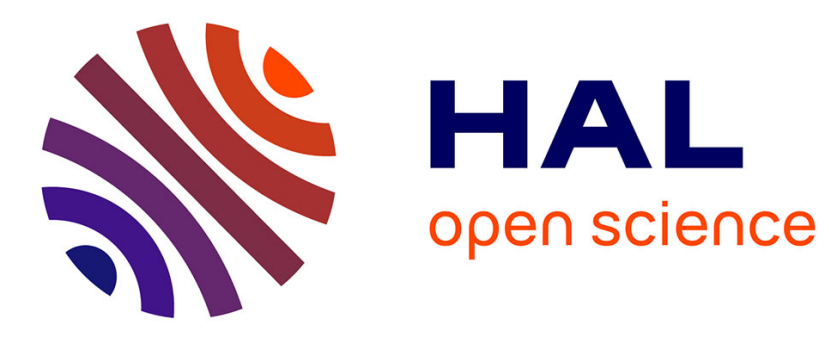

\title{
Logics of Reasonable Information Sources
}

Arnon Avron, Jonathan Ben-Naim, Beata Konikowska

\section{To cite this version:}

Arnon Avron, Jonathan Ben-Naim, Beata Konikowska. Logics of Reasonable Information Sources. 40th IEEE International Symposium on Multiple-Valued Logic (ISMVL 2010), Artificial Intelligence Research Institute of the Spanish National Research Council (IIIA-CSIC); University of Barcelona; Autonomous University of Barcelona; University of Lleida, May 2010, Barcelone, Spain. 10.1109/ISMVL.2010.19 . hal-03092778

\section{HAL Id: hal-03092778 https://hal.science/hal-03092778}

Submitted on 6 Jan 2021

HAL is a multi-disciplinary open access archive for the deposit and dissemination of scientific research documents, whether they are published or not. The documents may come from teaching and research institutions in France or abroad, or from public or private research centers.
L'archive ouverte pluridisciplinaire HAL, est destinée au dépôt et à la diffusion de documents scientifiques de niveau recherche, publiés ou non, émanant des établissements d'enseignement et de recherche français ou étrangers, des laboratoires publics ou privés. 


\title{
Logics of Reasonable Information Sources
}

\author{
Arnon Avron, Jonathan Ben-Naim and Beata Konikowska
}

\begin{abstract}
Extending the work of Belnap [3], we continue our research on ESP structures, consisting of information sources and a processor which collects information from the sources, and develops it further using certain coherent rules. We examine the case of reasonable sources which provide coherent information, characterize the logic of a single reasonable source, and prove that the logics generated by ESP structures with reasonable sources coincide with the general source-processor logics described in our previous work. However, we show that, unlike the case of arbitrary sources, there are processor valuations in general ESP structures which cannot be obtained from any finite number of valuations defined by reasonable sources.
\end{abstract}

\section{Introduction}

The development of new network technology allows several agents to access and update large knowledge bases, sometimes simultaneously. The process of combining information from different sources and drawing conclusions from it is very complex, especially in case of a need to deal with contradictory information.

This problem was tackled first by Belnap in [3], and his framework was extended by Carnielli and LimaMarques in [4]. Both works considered just the simple case of sources providing information only about atomic formulas of some logical language (which corresponds to the case of simple relational databases). This does not capture all the situations encountered in practice. Thus knowledge bases and disjunctive databases can provide information also about complex formulas.

A more general case was considered for the first time in [2]. The framework investigated there consists of a processor and a set of sources. A source may provide the processor with information about arbitrary formulas of the classical propositional language. To each formula $\psi$, it either assigns 1 to say that $\psi$ is true, 0 to say it is false, or nothing ("I") if it has no information about $\psi$. Thus each source in fact uses 3 truthvalues. In contrast (following Belnap's suggestion) the processor uses four truth-values, representing all possible combinations of the information on a formula that the processor can obtain from the sources:

$$
\mathbf{t}=\{1\}, \mathbf{f}=\{0\}, \top=\{0,1\}, \perp=\emptyset
$$

Here $\mathbf{t}$ and $\mathbf{f}$ correspond roughly to the classical truth values, while $T$ represents inconsistent information, and $\perp$ - lack of information.

The processor uses the information provided by the sources to assign the above truth-values to formulas. The assignment is carried out in two stages:

- In the first stage, the processor collects the information from the sources according to some strategy. The most basic strategy is the existential one, in which the processor initially includes a value $x \in\{0,1\}$ in the value of a formula $\psi$ iff some source assigns $x$ to $\psi$ (In the universal strategy, this happens if so do all the sources.). However, the processor can also base its decision on the opinion of the majority of sources, or of some subset of preferred sources, or use some other strategy.

- In the second stage, the processor expands the subset of $\{0,1\}$ which was initially assigned to a formula in order to close it under certain coherence constraints. Here the crucial assumption made in [2] is that the final assignment $v$ developed by the processor should include everything that can be derived from the classical truth tables without assuming consistency or full knowledge. Thus $0 \in v(\varphi \vee \psi)$ iff $0 \in v(\varphi) \cap v(\psi)$, and $1 \in v(\varphi \vee \psi)$ if $1 \in v(\varphi) \cup v(\psi)$. On the other hand it is possible that $1 \in v(\varphi \vee \psi)$ even if $1 \notin v(\varphi) \cup v(\psi)$. Moreover, because of the possibility of inconsistency, it can also happen that $1 \notin v(\varphi)$ even if $1 \in v(\varphi \vee \psi)$ and $0 \in v(\psi)$.

Now Dunn-Belnap's famous 4-valued matrix, which is used in Belnap's approach, is obtained in this framework if the processor collects information according to the existential strategy, and the sources provide information about atomic formulas only. However, if a source is allowed to provide information about compound formulas too, the above coherence constraints 
mean that the processor respects a certain 4-valued Nmatrix (non-deterministic matrix, see [1]), described in [2] and presented later on in this paper.

The framework developed in [2] has one main defect: While it imposes coherence constraints on the processor, it imposes none on the sources. One result of this, proved in [2], is that as long as there are at least two different sources, the number of sources has no effect on the resulting logic. In this paper, we consider a more realistic framework, and assume that the sources are also coherent. Moreover: unlike the processor, each source should be expected to be consistent. These assumptions mean that the sources should respect an Nmatrix too, but this time it is a 3 -valued Nmatrix with the truth-values $\{0,1, I\}$, where I represents absence of information. The said Nmatrix is more deterministic than the one used by the processor. Thus, according to the source Nmatrix it is impossible to assign 1 to $\varphi \vee \psi$ and 0 to $\psi$ without assigning 1 to $\varphi$. In fact, the interpretation of $\vee$ in this Nmatrix is like in Kleene's 3 -valued matrix, except that $\mathrm{I} \widetilde{V} \mathrm{I}=\{\mathrm{I}, 1\}$.

We shall call a source respecting the abovementioned Nmatrix reasonable. In the paper we present the logic of a single reasonable source. Then we show that if each source in a source-processor structure is reasonable, and the processor uses the existential strategy, then the resulting logics do depend in an essential way on the number of sources in the structure, and if no bound is imposed on that number, the logic coincides with the most general source-processor logic developed in [2]. This shows that, from the logical viewpoint, limiting attention to reasonable sources does not involve any loss of generality. Finally, we give new characterizations for the basic classes of source-processor structures examined in [2].

\section{Preliminaries}

We begin with introducing the basic notions connected with the information sources framework which will be employed throughout the paper. For the sake of simplicity, we assume that the formulas of classical propositional logic on which the sources give information are built using disjunction and negation only. Accordingly, all the matrices, Nmatrices and proof systems are presented here using the latter two connectives only. However, this does not cause any loss of generality compared to [2], where conjunction was also used, as in all the considered cases conjunction can be handled analogously to disjunction. Alternatively, it can be expressed in terms of disjunction and negation using De Morgan laws, with the same results.

\subsection{General source-processor framework}

Definition 2.1 Let $\mathcal{A}$ and $\mathcal{F}$ be the set of all atomic formulas and the set of all formulas, respectively, of the language $L_{C}$ of propositional classical logic with connectives limited to negation and disjunction only.

- By a source valuation we mean a function $s: \mathcal{F} \rightarrow$ $\{0,1, I\}^{1}$.

- By a processor-type valuation we mean a function $v: \mathcal{F} \rightarrow \mathcal{P}(\{0,1\})$.

- By a standard existential ${ }^{2}$ source-processor structure, shortly: ESP structure, we mean a tuple $\mathcal{S}=\langle S, g, d\rangle$, where:

1. $S$ is a non-empty set of source valuations;

2. $g$ is the processor-type valuation such that, for any $\varphi \in \mathcal{F}$ and any $x \in\{0,1\}$

$$
x \in g(\varphi) \text { iff } \exists s \in S . s(\varphi)=x
$$

3. $d$ is the minimal processor-type valuation satisfying the following conditions:

(d0) $g(\varphi) \subseteq d(\varphi)$ for every formula $\varphi$;

(d1) $0 \in d(\neg \varphi)$ iff $1 \in d(\varphi)$;

(d2) $1 \in d(\neg \varphi)$ iff $0 \in d(\varphi)$;

(d3) $1 \in d(\varphi \vee \psi)$ if $1 \in d(\varphi)$ or $1 \in d(\psi)$;

$(\mathrm{d} 4) 0 \in d(\varphi \vee \psi)$ iff $0 \in d(\varphi)$ and $0 \in d(\psi)$

- Conditions (d1)-(d4) in Pt. 3 are called the standard coherence conditions for $L_{C}$, and the minimal valuation $d$ specified there is called the processor valuation corresponding to the ESP-structure $\mathcal{S}$.

- A processor-type valuation $v$ is called an ESP processor valuation if there exists an ESP-structure $\mathcal{S}=\langle S, g, d\rangle$ such that $v=d$, i.e. $v$ is the processor valuation corresponding to $\mathcal{S}$.

It can be seen that formulas of $L_{C}$ do not have sufficient expressive power for describing some important facts regarding the source-processor structures. First, there is no way to express the fact that a certain formula $\varphi$ is not true, (i.e. $1 \notin d(\varphi)$ ), or to express disjunctive knowledge of the form "one of the formulas $\varphi$ and $\psi$ is known to be true" (i.e. either $1 \in d(\varphi)$ or $1 \in d(\psi))$. Second, $L_{C}$ does not possess an implication connective corresponding to the intended consequence relation. To compensate for this, as the mechanism for reasoning about source-processor structures

1 In [2] we used partial functions from $\mathcal{F}$ to $\{0,1\}$. The formulation used here is equivalent, with I representing an undefined value of such a function.

2 Other variants of processor strategies were mentioned and briefly discussed in [2]. 
we will use Gentzen-type sequents, which can be used to express the above two types of knowledge, and which provide a non-nestable version of implication as well.

\section{Definition 2.2}

By a sequent we mean a structure of the form $\Gamma \Rightarrow \Delta$, where $\Gamma$ and $\Delta$ are finite sets of formulas. The set of all sequents in the language $L_{C}$ is denoted by Seq.

Each source-processor structure generates a satisfaction relation on formulas in $\mathcal{F}$ and sequents in $S e q$, determined by the final processor valuation $d$ :

Definition 2.3 A source-processor $\mathcal{S}=\langle S, g, d\rangle$ is a model of (satisfies):

- a formula $\varphi \in \mathcal{F}$, in symbols $\models_{\mathcal{S}} \varphi$, iff $1 \in d(\varphi)$,

- a sequent $\Sigma=\Gamma \Rightarrow \Delta$, in symbols $\models_{\mathcal{S}} \Sigma$, iff either $\mathcal{S}$ is a model of some formula in $\Delta$, or it is not a model of some formula in $\Gamma$.

Accordingly, each source-processor structure or, more generally, a class of source-processor structures, induces the corresponding consequence relations:

Definition 2.4 Let $\mathcal{J}$ be a class of ESP structures.

- The formula consequence relation induced by $\mathcal{J}$ is the relation $\vdash_{\mathcal{J}}$ on $\mathcal{P}(\mathcal{F}) \times \mathcal{F}$ s.t. $T \vdash_{\mathcal{J}} \varphi$ if every $\mathcal{S} \in \mathcal{J}$ which is a model of $T$ is also a model of $\varphi$;

- The sequent consequence relation induced by $\mathcal{J}$ is the relation $\vdash_{\mathcal{J}}$ on $\mathcal{P}(S e q) \times S e q$ s.t. $Q \vdash_{\mathcal{J}} \Sigma$ if every model $\mathcal{S} \in \mathcal{J}$ of $Q$ is also a model of $\Sigma$.

Note that for the sake of simplicity, we use the same symbol for the formula consequence and sequent consequence relations. However, it will be always clear from the context which relation we actually have in mind.

Fact 2.1 For $F \subseteq \mathcal{F}$ and $\varphi \in \mathcal{F}$ :

- If $F$ is finite, then $F \vdash_{\mathcal{J}} \varphi$ iff $\vdash_{\mathcal{J}}(F \Rightarrow \varphi)$.

- $F \vdash_{\mathcal{J}} \varphi$ iff $\{\Rightarrow \psi \mid \psi \in F\} \vdash_{\mathcal{J}}(\Rightarrow \varphi)$.

\subsection{Non-deterministic Matrices}

Since formalization of the source-processor framework in the case when the sources can also provide information about complex formulas requires the use of Nmatrices, we shall now quote the relevant notions.

\section{Definition 2.5}

1. A non-deterministic matrix (Nmatrix) for a propositional language $\mathcal{L}$ is a tuple $\mathcal{M}=\langle\mathcal{V}, \mathcal{D}, \mathcal{O}\rangle$, where:

(a) $\mathcal{V}$ is a non-empty set of truth values.

(b) $\mathcal{D}$ is a non-empty proper subset of $\mathcal{V}$. (c) For every $n$-ary connective $\diamond$ of $\mathcal{L}, \mathcal{O}$ includes an $n$-ary function $\widetilde{\diamond}: \mathcal{V}^{n} \rightarrow 2^{\mathcal{V}}-\{\emptyset\}$.

2. Let $\mathcal{W}$ be the set of formulas of $\mathcal{L}$. A (legal) valuation in an Nmatrix $\mathcal{M}$ is a function $v: \mathcal{W} \rightarrow \mathcal{V}$ such that, for every $n$-ary connective $\diamond$ of $\mathcal{L}$ and every $\psi_{1}, \ldots, \psi_{n} \in \mathcal{W}$ :

$$
v\left(\diamond\left(\psi_{1}, \ldots, \psi_{n}\right)\right) \in \widetilde{\diamond}\left(v\left(\psi_{1}\right), \ldots, v\left(\psi_{n}\right)\right)
$$

3. A valuation $v$ in an Nmatrix $\mathcal{M}$ is a model of:

- a formula $\psi$ in $\mathcal{M}\left(v \models{ }^{\mathcal{M}} \psi\right)$ if $v(\psi) \in \mathcal{D}$.

- a set $T \subseteq \mathcal{W}$ in $\mathcal{M}\left(v \models^{\mathcal{M}} T\right)$ if $v \models^{\mathcal{M}} \psi$ for all $\psi \in T$.

- a sequent $\Sigma=\Gamma \Rightarrow \Delta\left(v \models^{\mathcal{M}} \Sigma\right)$ iff either $v \models^{\mathcal{M}} \psi$ for some $\psi \in \Delta$, or $v \not \models^{\mathcal{M}} \psi$ for some $\psi \in \Gamma$.

4. The formula consequence relation induced by $\mathcal{M}$ is the relation $\vdash_{\mathcal{M}}$ on $\mathcal{P}(\mathcal{W}) \times \mathcal{W}$ such that $T \vdash_{\mathcal{M}} \varphi$ if every model of $T$ in $\mathcal{M}$ is also a model of $\varphi$.

5. The sequent consequence relation induced by $\mathcal{M}$ is the relation $\vdash_{\mathcal{M}}$ on $\mathcal{P}(S e q) \times S e q$ such that: $\forall Q \subseteq S e q \forall \Sigma \in S e q, Q \vdash_{\mathcal{M}} \Sigma$ iff, for every valuation $v$ in $\mathcal{M}, v \models{ }^{\mathcal{M}} Q$ implies $v \models^{\mathcal{M}} \Sigma$.

Note that the formula and sequent consequence relations are bound by the relationships given in Fact 2.1.

\subsection{Main previous results}

In [2] we examined some basic variants of sourceprocessor structures, and characterized the logic corresponding to those structures. The fundamental result, extending Belnap's work, was the characterization of the most general source-processor logic, generated by the class of ESP structures in which the processor uses the existential strategy, and the sources can provide information about complex formulas too.

Consider the four-valued Nmatrix $\mathcal{M}_{I}^{4}=\langle\mathcal{V}, \mathcal{D}, \mathcal{O}\rangle$ with $\mathcal{V}=\{\mathbf{f}, \perp, \top, \mathbf{t}\}, \mathcal{D}=\{\top, \mathbf{t}\}, \mathcal{O}=\{\widetilde{\neg}, \widetilde{V}\}$, and the following interpretations of the connectives:

\begin{tabular}{c|cc|cccc}
$\widetilde{\neg}$ & & $\widetilde{V}$ & $\mathbf{f}$ & $\perp$ & $\top$ & $\mathbf{t}$ \\
\hline $\mathbf{f}$ & $\{\mathbf{t}\}$ & $\mathbf{f}$ & $\{\mathbf{f}, \top\}$ & $\{\mathbf{t}, \perp\}$ & $\{\top\}$ & $\{\mathbf{t}\}$ \\
$\perp$ & $\{\perp\}$ & $\perp$ & $\{\mathbf{t}, \perp\}$ & $\{\mathbf{t}, \perp\}$ & $\{\mathbf{t}\}$ & $\{\mathbf{t}\}$ \\
$\top$ & $\{\top\}$ & $\top$ & $\{\top\}$ & $\{\mathbf{t}\}$ & $\{\top\}$ & $\{\mathbf{t}\}$ \\
$\mathbf{t}$ & $\{\mathbf{f}\}$ & $\mathbf{t}$ & $\{\mathbf{t}\}$ & $\{\mathbf{t}\}$ & $\{\mathbf{t}\}$ & $\{\mathbf{t}\}$
\end{tabular}

In [2] we proved implicitly the following result:

Theorem 2.1 The set of processor valuations obtained in standard ESP structures coincides with the set of legal valuations in the Nmatrix $\mathcal{M}_{I}^{4}$. 
An immediate consequence of that result was the following characterization of the most general sourceprocessor logic:

Corollary 2.1 The logic generated by all ESP structures is identical with the logic generated by the Nmatrix $\mathcal{M}_{I}^{4}$, i.e., both the formula consequence relation and the sequent consequence relation generated by the class of all ESP structures and by the Nmatrix $\mathcal{M}_{I}^{4}$ are respectively identical.

In addition, we obtained the following characterizations of the logics corresponding to three special cases of the general source-processor framework for the existential strategy of the processor:

\section{Theorem 2.2}

- The logic generated by the class of all ESP structures in which the sources provide information about atomic formulas only is Dunn-Belnap's logic, generated by the 4-valued Dunn-Belnap's (deterministic) matrix.

- The logic generated by ESP structures in which the sources provide information about atomic formulas only, and taken together provide some information about all such formulas, is D'Ottaviano and da Costa's (deterministic) 3-valued basic paraconsistent logic [6].

- The logic $\mathcal{L}_{I}^{3}$ generated by ESP structures in which the sources taken together provide some information about all atomic formulas coincides with the logic generated by the 3-valued Nmatrix $\mathcal{M}_{I}^{3}=\langle\mathcal{V}, \mathcal{D}, \mathcal{O}\rangle$, where $\mathcal{V}=\{\mathbf{f}, \top, \mathbf{t}\}, \mathcal{D}=\{\top, \mathbf{t}\}, \mathcal{O}=\{\widetilde{\neg}, \widetilde{V}\}$, and the connectives are interpreted as follows:

\begin{tabular}{c|c}
$\widetilde{\neg}$ & \\
\hline $\mathbf{f}$ & $\{\mathbf{t}\}$ \\
$\top$ & $\{\top\}$ \\
$\mathbf{t}$ & $\{\mathbf{f}\}$
\end{tabular}

\begin{tabular}{c|ccc}
$\widetilde{V}$ & $\mathbf{f}$ & $T$ & $\mathbf{t}$ \\
\hline $\mathbf{f}$ & $\{\mathbf{f}, \top\}$ & $\{\top\}$ & $\{\mathbf{t}\}$ \\
$\top$ & $\{\top\}$ & $\{\top\}$ & $\{\mathbf{t}\}$ \\
$\mathbf{t}$ & $\{\mathbf{t}\}$ & $\{\mathbf{t}\}$ & $\{\mathbf{t}\}$
\end{tabular}

\section{A reasonable source and its logic}

\subsection{Behaviour of a reasonable source}

As explained in the introduction, particular attention should be paid to reasonable sources. These are sources which when providing information extend the knowledge they have with all consequences that can be derived from it based on classical truth tables, but without assuming completeness of information. In addition, a reasonable source should not provide inconsistent information. Thus, e.g., a source $s$ is not reasonable if $s(\varphi)=1$ but $s(\varphi \vee \psi)=\mathrm{I}$. On the other hand, for a reasonable source $s, s(\varphi \vee \psi)=0$ must imply $s(\varphi)=s(\psi)=0$.
One way to ensure this is to make the source satisfy the analogues of the coherence conditions (d1)-(d4) imposed on the processor in Def. 2.1 with membership replaced by equality:

Definition 3.1 An information source $s$ is called weakly coherent iff, for any $\varphi \in \mathcal{F}$, it satisfies the following conditions:

(s1) $s(\neg \varphi)=0$ iff $s(\varphi)=1$;

$(\mathrm{s} 2) s(\neg \varphi)=1$ iff $s(\varphi)=0$;

(s3) $s(\varphi \vee \psi)=1$ if $s(\varphi)=1$ or $s(\psi)=1$;

(s4) $s(\varphi \vee \psi)=0$ iff $s(\varphi)=0$ and $s(\psi)=0$

Unfortunately, the conditions are now too weak, for Condition (s3) does not prevent a source $s$ from assigning 1 to $\varphi \vee \psi$ even if $s(\varphi)=0$ and $s(\psi)=\mathrm{I}$ while a reasonable source should be able to infer from $s(\varphi \vee \psi)=1$ and $s(\varphi)=0$ that $s(\psi)=1^{3}$. To remedy this, we might replace the implication in (s3) by equivalence, obtaining the following definition:

Definition 3.2 An information source $s$ is called strongly coherent iff, for any $\varphi \in \mathcal{F}$, it satisfies Conditions (s1), (s2), (s4) of Def. 3.1, and:

(s3') $s(\varphi \vee \psi)=1$ iff $s(\varphi)=1$ or $s(\psi)=1$.

The latter conditions mean that the source observes the 3-valued Kleene Matrix $\mathcal{M}_{K}^{3}=\langle\mathcal{V}, \mathcal{D}, \mathcal{O}\rangle$, with $\mathcal{V}=\{1,0, \mathrm{I}\}, \mathcal{D}=\{1\}, \mathcal{O}=\{\widetilde{\neg}, \widetilde{V}\}$ and the following interpretations of the connectives:

\begin{tabular}{l|l}
$\simeq$ & \\
\hline 0 & 1 \\
1 & 0 \\
$\mathrm{I}$ & $\mathrm{I}$
\end{tabular}

\begin{tabular}{c|ccc}
$\widetilde{V}$ & 0 & 1 & $\mathrm{I}$ \\
\hline 0 & 0 & 1 & $\mathrm{I}$ \\
1 & 1 & 1 & 1 \\
$\mathrm{I}$ & $\mathrm{I}$ & 1 & $\mathrm{I}$
\end{tabular}

However, Condition (s3) is overly restrictive, compared to the behaviour we require from a reasonable source $s$. Namely, we do not rule out the possibility that a source $s$ might know that $\varphi \vee \psi$ is true without knowing either that $\varphi$ is true or that $\psi$ is true. This can happen when $s$ does not know the classical values of $\varphi$ and $\psi$ - i.e., when $s(\varphi)=s(\psi)=\mathrm{I}$.

Hence, instead of the Kleene matrix, a reasonable source should really obey an Nmatrix obtained out of the Kleene matrix by replacing $\mathrm{I}$ as the value of I $\widetilde{V} \mathrm{I}$ with the set $\{1, I\}$, and all the other entries - by the

3 Note that such a coherence condition, in opposition to the preceding ones, cannot be required of the processor - for it might receive inconsistent information from the sources. 
corresponding singletons sets. This results in the Nmatrix $\mathcal{M}_{r}^{3}=\langle\mathcal{V}, \mathcal{D}, \mathcal{O}\rangle$, with $\mathcal{V}, \mathcal{D}$ as above, and the following interpretations of negation and disjunction:

\begin{tabular}{l|l}
$\simeq$ & \\
\hline 0 & $\{1\}$ \\
1 & $\{0\}$ \\
$\mathrm{I}$ & $\{\mathrm{I}\}$
\end{tabular}

\begin{tabular}{c|ccc}
$\widetilde{V}$ & 0 & 1 & $\mathrm{I}$ \\
\hline 0 & $\{0\}$ & $\{1\}$ & $\{\mathrm{I}\}$ \\
1 & $\{1\}$ & $\{1\}$ & $\{1\}$ \\
$\mathrm{I}$ & $\{\mathrm{I}\}$ & $\{1\}$ & $\{\mathrm{I}, 1\}$
\end{tabular}

Thus we define a reasonable source as follows:

Definition 3.3 An information source $s$ is called reasonable if $s$ is a legal valuation in the Nmatrix $\mathcal{M}_{r}^{3}$.

\subsection{Logic of a single reasonable source}

By Def. 3.3, the logic defined by a single reasonable source is the logic generated by the the Nmatrix $\mathcal{M}_{r}^{3}$.

Definition 3.4 The sequent calculus $\mathcal{C}_{r}^{3}$ :

\section{Axioms:}

(A1) $\varphi \Rightarrow \varphi$

$$
\text { (A2) } \varphi, \neg \varphi \Rightarrow
$$

Structural inference rules: Weakening, Cut Logical inference rules:

$$
\begin{aligned}
& \frac{\Gamma, \varphi \Rightarrow \Delta}{\Gamma, \neg \neg \varphi \Rightarrow \Delta} \\
& \frac{\Gamma \Rightarrow \Delta, \varphi}{\Gamma \Rightarrow \Delta, \neg \neg \varphi} \\
& \frac{\Gamma \Rightarrow \Delta, \neg \varphi \quad \Gamma, \psi \Rightarrow \Delta}{\Gamma, \varphi \vee \psi \Rightarrow \Delta} \\
& \frac{\Gamma \Rightarrow \Delta, \neg \psi \quad \Gamma, \varphi \Rightarrow \Delta}{\Gamma, \varphi \vee \psi \Rightarrow \Delta} \\
& \frac{\Gamma, \neg \varphi, \neg \psi \Rightarrow \Delta}{\Gamma, \neg(\varphi \vee \psi) \Rightarrow \Delta} \quad \frac{\Gamma \Rightarrow \Delta, \neg \varphi \quad \Gamma \Rightarrow \Delta, \neg \psi}{\Gamma \Rightarrow \Delta, \neg(\varphi \vee \psi)} \\
& \begin{array}{c}
\Gamma, \Delta, \varphi, \psi \\
\Gamma \Rightarrow \Delta, \varphi \vee \psi
\end{array}
\end{aligned}
$$

Definition 3.5 Let $\underline{\mathcal{C}}_{r}^{3}$ be the calculus obtained from $\mathcal{C}_{r}^{3}$ by limiting the applications of the cut rule to formulas occurring in the premises of sequent derivations. In other words: If $S=\left\{\Gamma_{1} \Rightarrow \Delta_{1}, \ldots, \Gamma_{n} \Rightarrow \Delta_{n}\right\}$ then $S \vdash_{\underline{\mathcal{C}}_{r}^{3}} \Sigma$ if there is a proof of $\Sigma$ from $S$ in $\mathcal{C}_{r}^{3}$ where all cuts are made on formulas in $\bigcup_{i=1}^{n}\left(\Gamma_{i} \cup \Delta_{i}\right)$ (in particular: $\vdash_{\underline{\mathcal{C}}_{r}^{3}} \Sigma$ iff $\Sigma$ has a cut-free proof in $\mathcal{C}_{r}^{3}$ ).

Theorem 3.1 The calculus $\underline{\mathcal{C}}_{r}^{3}$ is finitely strongly sound and complete for $\vdash_{\mathcal{M}_{r}^{3}}$, i.e., for any finite set of sequents $S \subseteq$ Seq and any sequent $\Sigma \in S e q, S \vdash_{\mathcal{M}_{r}^{3}} \Sigma$ iff $S \vdash_{\underline{\mathcal{C}}_{r}^{3}} \Sigma$.

Proof (sketch) As it is easy to see that $\mathcal{C}_{r}^{3}$ is strongly sound for $\vdash_{\mathcal{M}_{r}^{3}}$, it suffices to prove the strong completeness of $\underline{\mathcal{C}}_{r}^{3}$ for finite premise sets.

We argue by contradiction. Suppose that for a finite set of sequents $S$ and a sequent $\Sigma_{0}$ we have $S \vdash_{\mathcal{M}_{r}^{3}} \Sigma_{0}$, but $S \nvdash_{\mathcal{C}^{3}} \Sigma_{0}$. We construct a counter-valuation $v$ such that $v \models \mathcal{M}_{r}^{3} S$ but $v \not \forall^{\mathcal{M}_{r}^{3}} \Sigma_{0}$. Call a sequent $\Gamma \Rightarrow \Delta$ saturated if it is closed under the logical rules in $\underline{\mathcal{C}}_{r}^{3}$ applied backwards (e.g., if $\neg(\varphi \vee \psi) \in \Gamma$ then both $\neg \varphi$ and $\neg \psi$ are in $\Gamma$, while if $\neg(\varphi \vee \psi) \in \Delta$ then either $\neg \varphi$ or $\neg \psi$ is in $\Delta$ ). To construct the valuation $v$, we construct inductively an extension $\Gamma^{*} \Rightarrow \Delta^{*}$ of $\Sigma_{0}$ which is a saturated sequent, and define $v$ as follows:

- For any atomic $p, v(p)= \begin{cases}1 & \text { if } p \in \Gamma^{*} \\ 0 & \text { if } \neg p \in \Gamma^{*} \\ \mathrm{I} & \text { otherwise }\end{cases}$

- For any formulas $\varphi, \psi: v(\neg \varphi)= \begin{cases}1 & \text { if } v(\varphi)=0 \\ 0 & \text { if } v(\varphi)=1 \\ \text { I } & \text { otherwise }\end{cases}$

$$
v(\varphi \vee \psi)= \begin{cases}1 \quad \text { iff } v(\varphi)=1 \text { or } v(\psi)=1 \text { or } \\ & v(\varphi)=v(\psi)=\mathrm{I} \text { and }(\varphi \vee \psi) \in \Gamma^{*} \\ 0 & \text { iff } v(\varphi)=0 \text { and } v(\psi)=0 \\ \mathrm{I} \quad \text { otherwise }\end{cases}
$$

Then it can be shown that $v$ is a well-defined, legal valuation for $\mathcal{M}_{r}^{3}$. Moreover, we have $v \models \mathcal{M}_{r}^{3} S$ and $v \not \mathcal{M}_{r}^{3} \Sigma_{0}$, so $v$ is the desired counter-valuation.

As $\underline{\mathcal{C}}_{r}^{3}$ is a restriction of $\mathcal{C}_{r}^{3}$ and the full cut rule is sound for $\mathcal{M}_{r}^{3}$, Theorem 3.1 has the following immediate consequence:

Corollary 3.1 The calculus $\mathcal{C}_{r}^{3}$ is finitely sound and complete for $\vdash_{\mathcal{M}_{r}^{3}}$, and the cut rule is admissible in it.

Note that $\mathcal{C}_{r}^{3}$ can be obtained from the calculus $\mathcal{C}_{I}^{4}$ for the most general, 4-valued source-processor logic $\mathcal{L}_{I}^{4}$ given in [2] by replacing rule $(\vee \Rightarrow)$ of $\mathcal{C}_{I}^{4}$, which is not valid for $\mathcal{M}_{r}^{3}$, with the two $(\vee \Rightarrow)$-rules given here.

\section{Source-processor logics for ESP structures with reasonable sources}

Now we show that instead of ESP structures with arbitrary, possibly irrational sources, we can confine ourselves to ESP structures with reasonable sources.

Theorem 4.1 The logic $\mathcal{L}_{R}^{4}$ generated by the ESP structures in which all sources are reasonable is identical with the most general source-processor logic $\mathcal{L}_{I}^{4}$, defined by the class of all ESP structures with no coherence conditions imposed on the sources.

Proof (Sketch) As each processor valuation generated by reasonable sources is a legal processor valuation in the general 4-valued framework of Section 2.1, then $\mathcal{L}_{I}^{4} \subset \mathcal{L}_{R}^{4}$. To prove the converse inclusion, we show that each such general processor valuation $v$ can be obtained from valuations defined by reasonable sources. To this end, we split $v$ into a family of source valuations $S_{v}$ defined as follows: 
1. For any atomic formula $p \in \mathcal{A}$, we include in $S_{v}$ source valuations $s_{p}^{0}, s_{p}^{1}$ such that:

$$
\begin{aligned}
& s_{p}^{0}(p)=\left\{\begin{array}{ll}
0 & \text { if } 0 \in v(p) \\
\mathrm{I} & \text { otherwise }
\end{array} \quad s_{p}^{1}(p)= \begin{cases}1 & \text { if } 1 \in v(p) \\
\mathrm{I} & \text { otherwise }\end{cases} \right. \\
& \text { and } s_{p}^{i}(q)=\mathrm{I} \text { for } q \in \mathcal{A}, q \neq p, i=0,1 .
\end{aligned}
$$

2. For any formula $\psi=\alpha \vee \beta$ such that $1 \in v(\alpha \vee \beta)$, we include in $S_{v}$ a source valuation $s_{\psi}$ such that:

$$
\begin{gathered}
s_{\psi}(\alpha \vee \beta)=1, \quad s_{\psi}(\chi)=\mathrm{I} \text { for every proper } \\
\text { subformula } \chi \text { of } \alpha \vee \beta
\end{gathered}
$$

3. The above partial source valuations are extended to all formulas in $\mathcal{F}$ according to the truth tables of the Kleene matrix $\mathcal{M}_{K}^{3}$.

Then it can be shown that:

1. Each $s \in S_{v}$ represents a reasonable source.

2. The final processor valuation $d$ obtained out of the source valuations in $S_{v}$ according to Definition 2.1 coincides with $v$.

An analogous result holds for the logics generated by the special cases of ESP structures examined in [2]:

Proposition 4.1 Theorem 2.2 remains true for ESP structures containing reasonable sources only.

Despite Theorem 4.1 and Proposition 4.1, there is a crucial difference between the corresponding frameworks. In case of arbitrary sources, it is in fact enough to consider ESP structures with just two sources, for they generate the same logic as all ESP structures. However, the logic of ESP structures with reasonable sources cannot be generated by structures with a bounded finite number of sources:

Proposition 4.2 There are ESP processor valuations which cannot be obtained from any finite number of valuations defined by reasonable sources.

Proof Let $p_{0}, p_{1}, p_{2}, \ldots$ be an infinite sequence of atomic formulae, and define formulae $B_{1}, B_{2}, \ldots$ by:

- $B_{1}=p_{0} \vee p_{1}$;

- $B_{n+1}=\neg\left(B_{1} \vee B_{2} \vee \ldots \vee B_{n}\right) \vee p_{n+1}$ for $n=1,2, \ldots$, where $B_{1} \vee B_{2} \vee \ldots \vee B_{n}$ is left-associative.

We define the desired processor valuation $v$ by taking

$$
\begin{aligned}
& v\left(p_{k}\right)=\perp \text { for all } k \geq 0 ; \\
& v\left(B_{n}\right)=\mathbf{t}, \quad v\left(B_{1} \vee B_{2} \vee \ldots \vee B_{n}\right)=\mathbf{t}, \text { and } \\
& v\left(\neg\left(B_{1} \vee B_{2} \vee \ldots \vee B_{n}\right)\right)=\mathbf{f} \text { for all } n \geq 1
\end{aligned}
$$

and extending $v$ to other formulae according to the Dunn-Belnap matrix [3]. Then $v$ is a valid ESP processor valuation. Suppose now $S$ is a set of reasonable sources generating the valuation $v$. Then, as $v\left(p_{k}\right)=\perp$ for all $k \geq 0$, we must necessarily have $s\left(p_{k}\right)=\perp$ for all $k \geq 0$ and each $s \in S$. Hence if a reasonable source $s \in S$ assigns 1 to some $B_{n}$, then it assigns I to every $B_{k}$ such that $k>n-$ and so also to every $B_{k}$ such that $k<n$. This easily implies that for every $k$ we need a different source $s \in S$ that assigns 1 to $B_{k}$ to ensure that $v\left(B_{i}\right)=\mathbf{t}$. Hence $S$ must be infinite.

As the motivation for our work is handling information from arbitrarily many sources, then Proposition 4.2 clearly implies that structures based on reasonable sources are more adequate than those with arbitrary sources - for a framework reducible to twoelement structures is trivial from practical viewpoint.

\section{Conclusions and future work}

A natural next step in our research will be to extend the language of ESP structures with suitably interpreted quantifiers to obtain a first-order logic of sourceprocessor structures. Another direction will be considering a more realistic, "deductive" version of a reasonable source, which possesses information about a finite number of formulas only, but uses it to deduce information about other formulas.

Further, the problems considered in our work bear an obvious relationship to the works on on social choice, e.g. [5], where a group of individuals aggregate their individual judgments on some interconnected propositions into the corresponding collective judgment. Hence another direction of future work would be to try to apply our approach to the problems of social choice.

\section{References}

[1] A. Avron, and I. Lev, Non-deterministic Multiple-valued Structures, Journal of Logic and Computation, Vol. 15 (2005), 241-261.

[2] A. Avron, J. Ben-Naim, and B. Konikowska, Processing Information from a Set of Sources, in Towards Mathematical Philosophy, (D. Makinson, J. Malinowski, and H. Wansing, eds.), Trends in Logic 28, 165-186, Springer, 2009.

[3] N. D. Belnap, A useful four-valued logic, In G. Epstein and J. M. Dunn, editors, Modern Uses of MultipleValued Logic, 7-37. Reidel, Dordrecht, 1977.

[4] W. A. Carnielli and M. Lima.-Marques, Society semantics for multiple-valued logics, in Proc. of XII EBL, (Walter A. Carnielli and Itala M. L. D'Ottaviano, eds.), AMS Series Contemporary Mathematics, Volume 235, 33-52, 1999.

[5] F. Dietrich and C. List, Strategy-Proof Judgment Aggregation, Economics and Philosophy, vol.23, pp.269-300, 2007.

[6] I.M.L. D'Ottaviano and N.C.A. da Costa, Sur un problème de Jaśkowski. In Comptes Rendus de l'Académie des Sciences de Paris, Vol. 270 (1970), 1349-1353. 